\title{
Detection of a mosaic hidden behind a plaster layer by IR thermography
}

\author{
by A. MAZIOUD*, L. IBOS*, J. DUMOULIN** \\ * Université Paris-Est, CERTES, 61 avenue du Général De Gaulle, 94010 Créteil Cedex, France, \\ vincent.feuillet@u-pec.fr \\ ** Université Paris-Est, LCPC, Route de Bouaye, BP 4129, 44341 Bouguenais Cedex, France
}

\begin{abstract}
:
A feasibility study on thermal signature discrimination for mosaic hidden behind a plaster layer was realised using active infrared thermography. Particularly, influence of four types of joints width (from $1 \mathrm{~mm}$ to $4 \mathrm{~mm}$ ) between the elements of the mosaic made of polystyrene was investigated. Experimental results were compared to simulation results obtained with Fluent ${ }^{\mathrm{TM}}$. Two analysis approaches in active infrared thermography were tested: computation of contrast on one hand, and singular-value decomposition (SVD) of thermal image sequences. Images obtained from simulation results show that the presence of the mosaic can be directly detected. In experimental part, the presence of joints cannot be detected by a simple contrast as we suspected the plot of time dependence of absolute contrast. However the plot of time dependence of absolute contrast obtained from simulations results along two horizontal lines allows observing the presence of joints of width greater or equal to $2 \mathrm{~mm}$.
\end{abstract}

\section{Introduction}

The purpose of this study is to detect the presence of a mosaic hidden behind a layer of plaster by active infrared thermography. Active infrared thermography for the detection of defects has been used now for many years for non-destructive control of materials such as metals, composites and so on, as described in the literature [1].

The objective of this study was to evaluate to which extent this technique could be applied to this particular application. There is large variety of materials used for mosaic, from marble to wood. They might have different granular constitution and therefore different thermal properties. Their structures could be regular or non-regular. In this part we will particularly study the influence of joints width between the elements of the mosaic. Experimental results will be compared to simulation results obtained using Fluent ${ }^{\mathrm{TM}}$. Two analysis approaches in active infrared thermography were tested: computation of contrast on one hand, and singular-value decomposition (SVD) of thermal image sequences.

\section{Experimental measurements}

\subsection{Laboratory Sample description}

The sample is composed of 4 zones. Each zone consists of nine polystyrene parallelepipeds with $20 \mathrm{~mm} \times$ $20 \mathrm{~mm} \times 10 \mathrm{~mm}$ dimensions. They are spaced from neighbours by $1 \mathrm{~mm}$ of plaster joints in the first zone, $2 \mathrm{~mm}$ in the second zone, $3 \mathrm{~mm}$ in the third zone and $4 \mathrm{~mm}$ in the fourth zone. The parallelepipeds are fixed onto a mortar plate $300 \mathrm{~mm} \times 300 \mathrm{~mm}$ size and $10 \mathrm{~mm}$ thickness. Then, they are covered with a plaster layer of $8 \mathrm{~mm}$ thickness.

A schematic view of the sample considered during its manufacturing is presented in figure 1 . The first layer (front face) is made of plaster of dimension $300 \mathrm{~mm}$ by $300 \mathrm{~mm}$ on which we fixed the parallelepipeds made of extruded polystyrene. The Parallelepipeds elements are distributed on four zones. Zones 1 to 4 will correspond further in this article to joints width of $1 \mathrm{~mm}$ to $4 \mathrm{~mm}$ respectively. Each zone containing nine polystyrene elements is spaced of $80 \mathrm{~mm}$ from its neighbours. Five Thermocouples are placed in the sample to measure the evolution of the temperatures, four at the surface of joints and one placed in the center of the sample. Themocouple measurements will not be considered in this study. The second layer made of plaster with $10 \mathrm{~mm}$ of thickness is placed on the fill polystyrene parallelepipeds elements. Finally, the third layer (rear face) made of mortar is used to stiffen the sample.

\subsection{Experimental set-up}

The experimental set-up was developed in laboratory. A picture of the experimental set-up is presented in figure 2. Front face of the sample was heated using the square heating method using two halogen lamps of $500 \mathrm{~W}$. Thermal image recordings were performed during sample heating and subsequent cooling using a FLIR ${ }^{\odot}$ A325 long wave (7.5 $13 \mu \mathrm{m}$ ) un-cooled IRFPA camera. The distance between the camera lens and the sample surface was fixed to $75 \mathrm{~cm}$. In these experimental conditions, the spatial resolution in the thermal scene observed is approximately equal to $1 \mathrm{~mm}$, which match the width of the plaster joints in zone 1.

Heating duration was fixed to $90 \mathrm{~s}$. The total duration of one experiment was fixed to 1800 s. Experimental set-up was controlled by a program developed under Labview ${ }^{\odot}$. Heat pulse duration and intensity were monitored. Two sampling frequencies were used: $1 \mathrm{~Hz}$ and $4 \mathrm{~Hz}$. For the sampling frequency of $1 \mathrm{~Hz}$, each thermal image recorded 
corresponds to the average of 30 images acquired at the maximum sampling frequency $(60 \mathrm{~Hz})$. In the case of the use of a $4 \mathrm{~Hz}$ sampling frequency, each image recorded corresponds to the average of 8 images acquired at the maximum sampling frequency $(6 \mathrm{~Hz})$. Sample surface emissivity was fixed to 0.89 for the computation of apparent sample surface temperature.

A schematic description of the regions of interest which will be considered for the analysis of experimental data is presented in figure 3. Temperature profiles recorded at the center of the sample and at the center of each polystyrene "defect" zone will be considered first. Temperature profiles along rows and columns will also be considered. Finally, figure 3 presents the position of the four polystyrene zones (1 to 4 ) corresponding to joint widths increasing from 1 to 4 $\mathrm{mm}$ respectively to the field of view (FOV) of the camera.

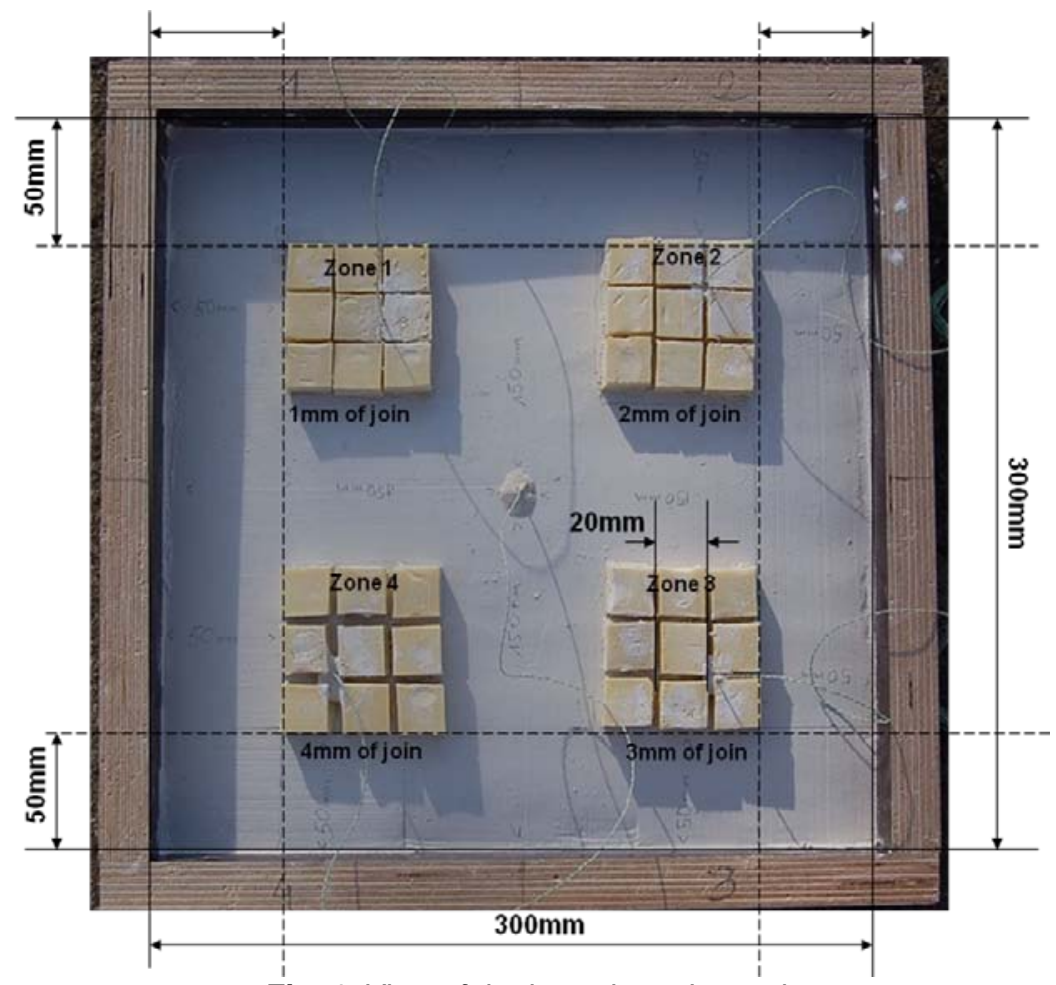

Fig. 1. View of the investigated sample

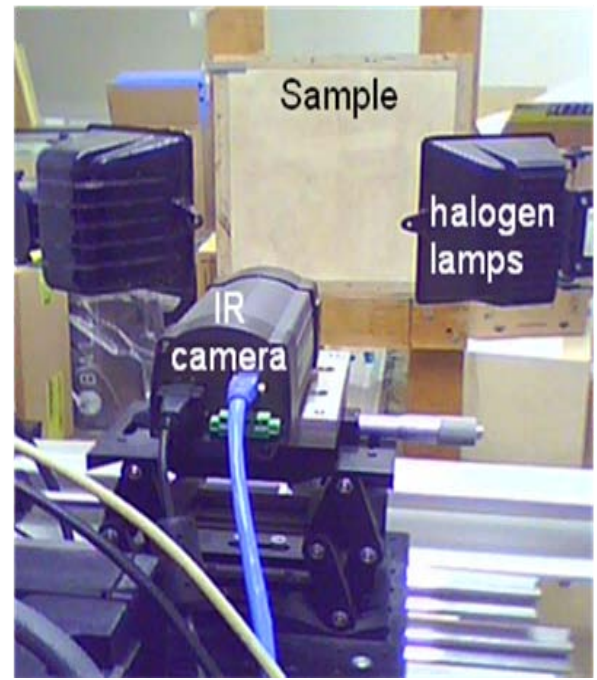

Fig 2. View of the experimental setup

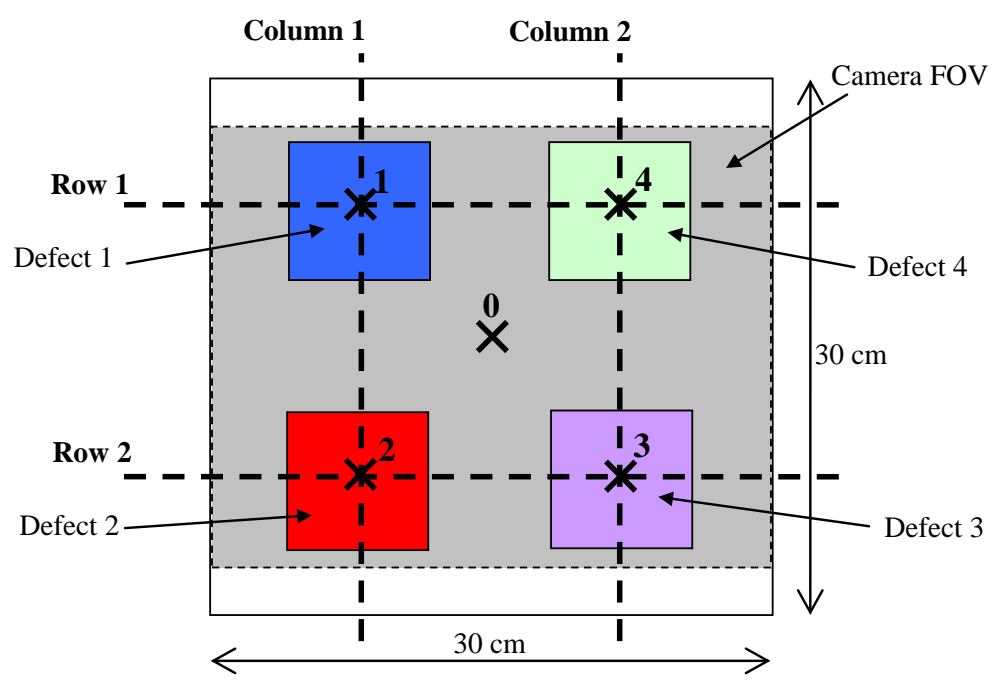

Fig 3. Position of mosaic "defects" (as seen by the infrared camera during experiments) and of regions of interest (Points, Rows, Columns) 


\section{Numerical Modeling}

A schematic description of the sample considered for numerical simulations is presented in figure 4 . The main differences with the manufactured and experimentally tested sample are the fact that the four polystyrene blocks are separated by a plaster joint of $1 \mathrm{~mm}$ width and that the dimension of polystyrene parallelepipeds are $10 \mathrm{~mm} \times 10 \mathrm{~mm} X$ $10 \mathrm{~mm}$. This choice allows reducing the sample volume considered for simulations and consequently the number of cells in the mesh grid and the computation time. Nevertheless, it also change ratio between plaster and polystyrene for each type of mosaic.

A 3D mesh of the sample was performed using Gambit software (see figure 5). Considering the complex geometry of the sample due to the variable gap between adjacent parallelepipeds, an unstructured tetrahedral mesh composed of 540,000 cells was used. Nodes are spaced by $0.1 \mathrm{~mm}$ from one to each other. The maximum skewness is about 0.74 . Numerical simulations were carried out using Fluent ${ }^{\mathrm{TM}}$. A heat flux density is applied to the front face for a given duration and the temperature field is calculated on the plaster front face during the heating phase and the subsequent cooling. The heating duration was chosen equal to $90 \mathrm{~s}$, and temperature field inside the sample was computed each second. A heating power density of $2620 \mathrm{~W} / \mathrm{m}^{2}$ was chosen, accordingly to previous results carried out using similar test bench except for the homogeneity caisson equipment [2]. The thermophysical properties of materials used are listed in table 1.

Boundary conditions were chosen as follows. First, we assumed that the sample is insulated on all its lateral faces. On the rear face of the sample, a constant global heat exchange coefficient of $10 \mathrm{~W} \cdot \mathrm{m}^{-2} \cdot \mathrm{K}^{-1}$ was used. A variable heat exchange coefficient $h$ was used to model heat exchanges on the sample front face, in order to take into account the front side temperature changes. This coefficient is defined as follows:

$$
h=h_{c}+h_{r}=1.24\left(T_{W}-T_{e n v}\right)^{1 / 4}+4 \sigma\left(\left(T_{W}+T_{e n v}\right) / 2\right)^{3}
$$

where $T_{\mathrm{w}}$ is the front face temperature, $\sigma$ is the Boltzmann constant and $T_{\text {env }}$ is the environment temperature, kept constant and equal to $293 \mathrm{~K}$. The initial temperature of the whole sample is $293 \mathrm{~K}$.

Table 1. Values of thermophysical properties of plaster, mortar and polystyrene used for numerical simulations

\begin{tabular}{|c|c|c|c|}
\hline Material & Density $\rho\left(\mathrm{kg} \cdot \mathrm{m}^{-3}\right)$ & Specific heat Cp $\left(\mathrm{J} \cdot \mathrm{kg}^{-1} \cdot \mathrm{K}^{-1}\right)$ & Thermal Conductivity $\mathrm{k}\left(\mathrm{W} \cdot \mathrm{m}^{-1} \cdot \mathrm{K}^{-1}\right)$ \\
\hline Polystyrene & 33 & 1450 & 0.05 \\
\hline Plaster & 1150 & 1000 & 0.57 \\
\hline Mortar & 2200 & 1000 & 1.4 \\
\hline
\end{tabular}

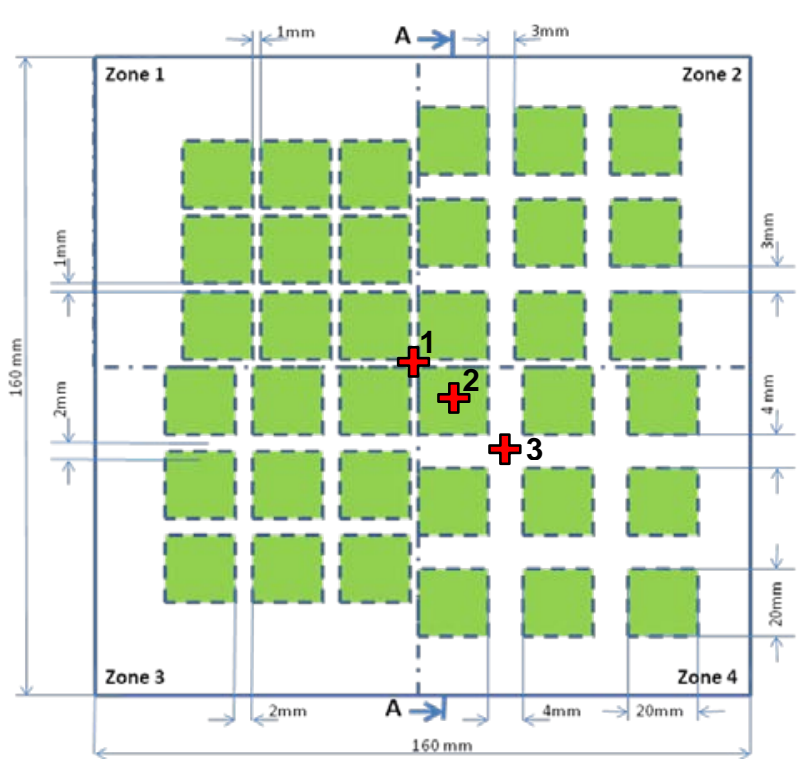

Fig. 4. Sample structure and dimensions
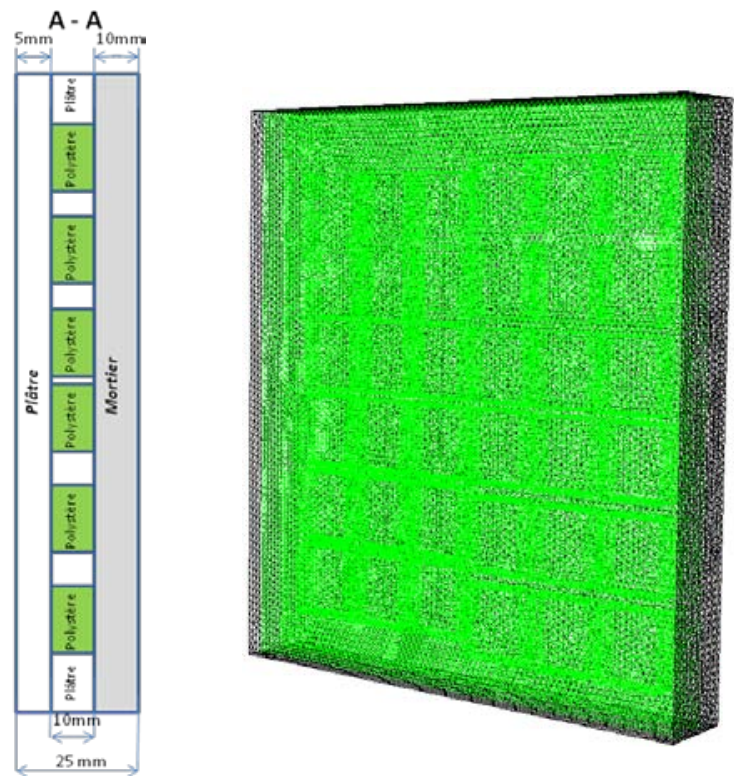

Fig. 5. 3D mesh of the sample 


\section{Results}

\subsection{Raw temperature results}

The evolution of the temperature recorded on the sample surface at the five points defined in figure 3 is presented in figure 6 . Numerical simulation results are presented in figure 7 for points located in equivalent area. The maximum increase of temperature at the end of heating phase is at least twice important for numerical simulations than for experiments. Such situation is probably due to a combination of two differences between experiment and simulation.. The first one is the difference of power density applied to surface during experiments and numerical simulation as no homogeneous dispositive was used for optical solicitation contrary to ref [2] and a different distance between object and lamps. The second one is that plaster is more reflective in the spectral range of the halogen lamps used than it is in the LWIR range where measurements are done. The highest temperatures during the cooling phase are obtained in the centre of the mosaic with joints of $1 \mathrm{~mm}$ width.

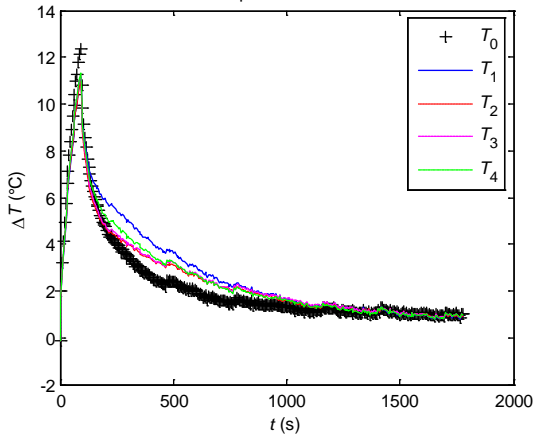

Fig. 6. Experimental temperature profiles in the centre of the sample $\left(T_{0}\right)$ and of each polystyrene mosaic ( $T_{1}$ to $\left.T_{4}\right)$

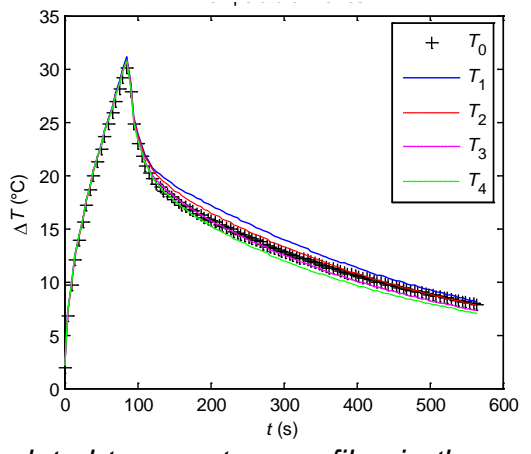

Fig. 7. Simulated temperature profiles in the centre of the sample $\left(T_{0}\right)$ and of each polystyrene mosaic ( $T_{1}$ to $\left.T_{4}\right)$

Absolute contrast thermal images calculated at 100 and $400 \mathrm{~s}$ after the end of heating phase are presented in figures 8 and 9 . Equivalent images calculated with thermal images retrieved from Fluent ${ }^{\mathrm{TM}}$ numerical simulations corresponding to the same experimental durations are presented in figures 10 and 11 . We remind that temperature values reported in these images correspond to temperature variations since the beginning of the experiment or simulation, as a cold image was subtracted to thermal images before plotting. Moreover, positions of the four mosaics are not the same for experimental and simulated images. For experimental images, positions are defined in figure 3 . For simulations joints of $1 \mathrm{~mm}$ are placed in the top left part of images, joints of $2 \mathrm{~mm}$ in the bottom left part, joints of $3 \mathrm{~mm}$ in the top right part and finally joints of $4 \mathrm{~mm}$ in the bottom right part.

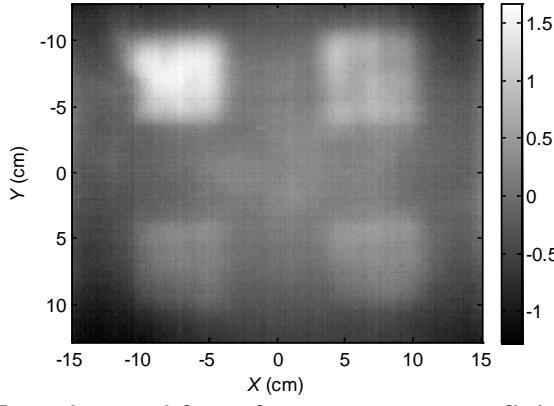

Fig. 8. Experimental front face temperature field recorded 100s after the end of heating

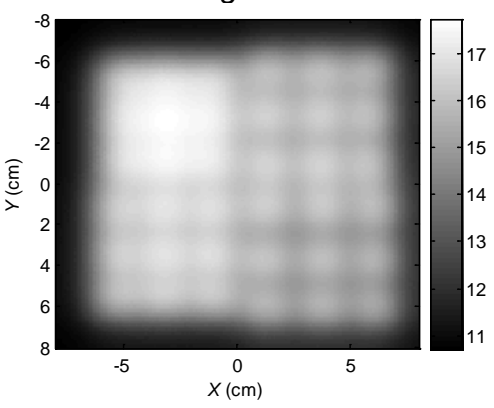

Fig. 10. Simulated front face temperature field 100s after the end of heating

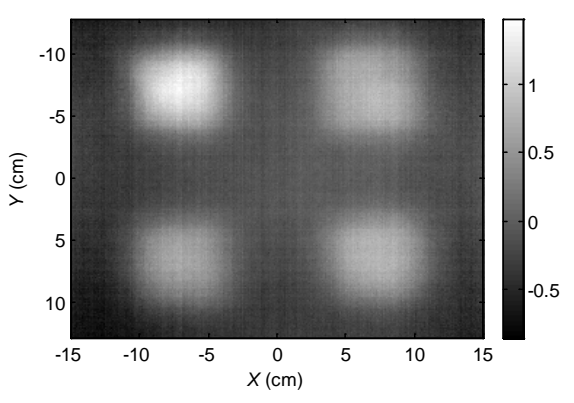

Fig. 9. Experimental front face temperature field recorded 400s after the end of heating

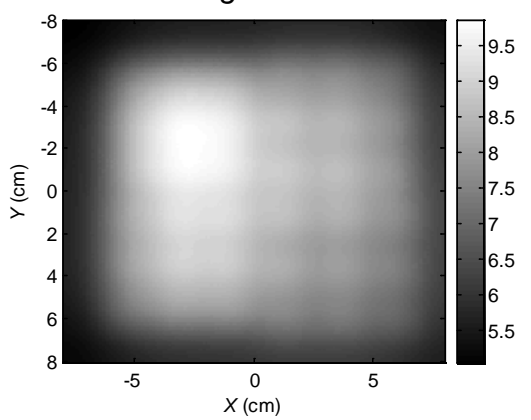

Fig. 11. Simulated front face temperature field 400 s after the end of heating 
Figures corresponding to experimental results allow observing the thermal signature of the four polystyrene mosaics, but it is not possible to observe the presence of plaster joints. Moreover, polystyrene mosaics of zones 1 and 4 seem to appear earlier than the two other ones. Images obtained from simulation results show that the presence of the mosaic can be directly detected. The structure of the mosaic also appears excepted when considering smallest joints.

\subsection{Contrast analysis}

Two different contrast computations were used: absolute contrast and standard contrast [1]. Absolute contrast is defined as the temperature difference between a defect zone and a sound area [1]:

$$
C_{a}(t)=T_{\text {def }}(t)-T_{\text {sound }}(t)
$$

The standard contrast is defined by the ratio between temperature variations in a defect zone and a sound region [1]:

$$
C_{S}(t)=\frac{T_{\text {def }}(t)-T_{\text {def }}(0)}{T_{\text {sound }}(t)-T_{\text {sound }}(0)}
$$

The problem in using these definitions is that defect and sound regions have to be defined before starting contrast computations. In this study, we chose to replace the temperature of the sound area by the mean temperature of the thermal image. This method was successfully tested for the detection of defects embedded into road pavement samples [2]. This method allows computing the contrast in each pixel of coordinates $(\mathrm{i}, \mathrm{j})$ without considering any defect or sound area. Thus, equations 2 and 3 can be rewritten as follows:

$$
\begin{array}{r}
C_{a}(i, j, t)=T(i, j, t)-\bar{T}(t) \\
C_{S}(i, j, t)=\frac{T(i, j, t)-T(i, j, 0)}{\bar{T}(t)-\bar{T}(0)}
\end{array}
$$

Absolute and standard contrast computation performed using experimental measurements data are presented in figures 12 and 13 respectively, considering the temperature variations at the centre of each polystyrene mosaic.

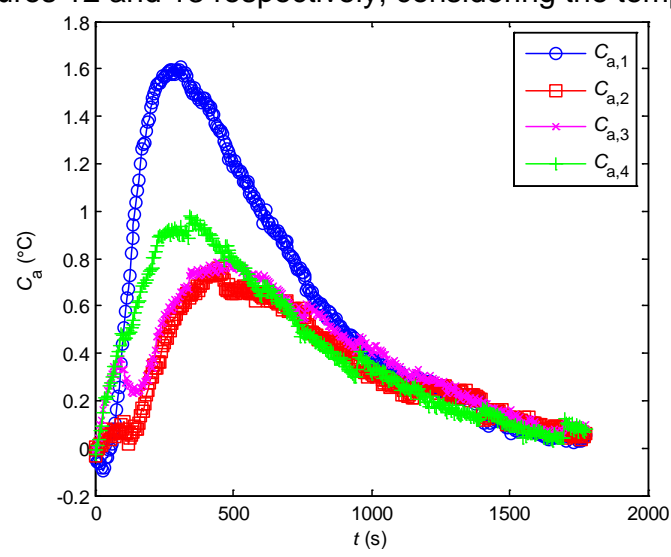

Fig. 12. absolute contrast computed at the centre of zones 1, 2, 3 and 4

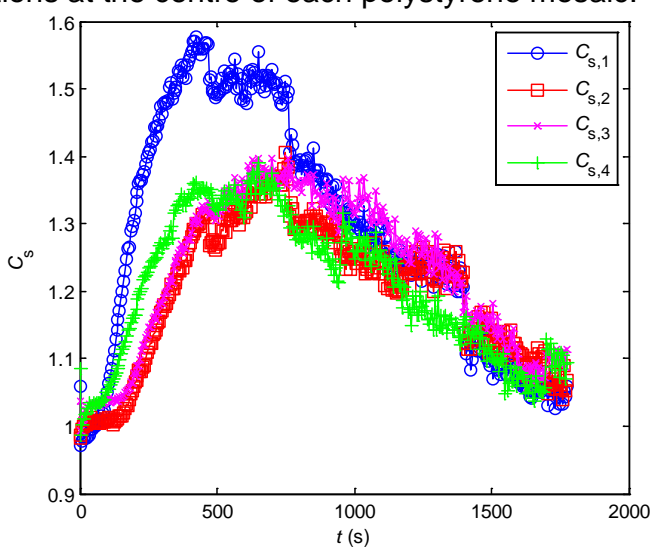

Fig. 13. standard contrast computed at the centre of zones 1, 2, 3 and 4

The absolute and standard contrast curves exhibit a peak whose maximum position and magnitude depend on the size of plaster joints. The greater value of maximum contrast is obtained for the analysis performed on the mosaic with joints of $1 \mathrm{~mm}$ width. For this same case the peak maximum is reached for a shorter time than for mosaics with larger joints. This may reflect the fact that the thermal resistance of the mosaic with $1 \mathrm{~mm}$ width joint is the greatest one. On the contrary, the absolute contrast obtained for the zone with joints of $4 \mathrm{~mm}$ width exhibits a peak whose amplitude is greater than the one obtained for mosaic with joints of 2 and $3 \mathrm{~mm}$. Moreover the maximum is reached for shorter experiment duration than for these same mosaics. Such unexpected result could be interpreted by a non-uniform heating of the sample. Indeed, this phenomenon can only be observed on absolute contrast. The standard contrast curves obtained for mosaics with plaster joints width of 2, 3 and $4 \mathrm{~mm}$ are quite similar. It is known that standard contrast computation is less sensitive to a non-uniform heating than absolute contrast. 
Absolute and standard contrasts computed along rows and columns defined in figure 3 are plotted as a function of time in figures 14 and 15. Results plotted in these figures tend to confirm previous observation. Moreover, these plots also confirm that the presence of joints cannot be detected by a simple contrast analysis as any signature corresponding to the presence of joints is observable on figures 14 and 15.
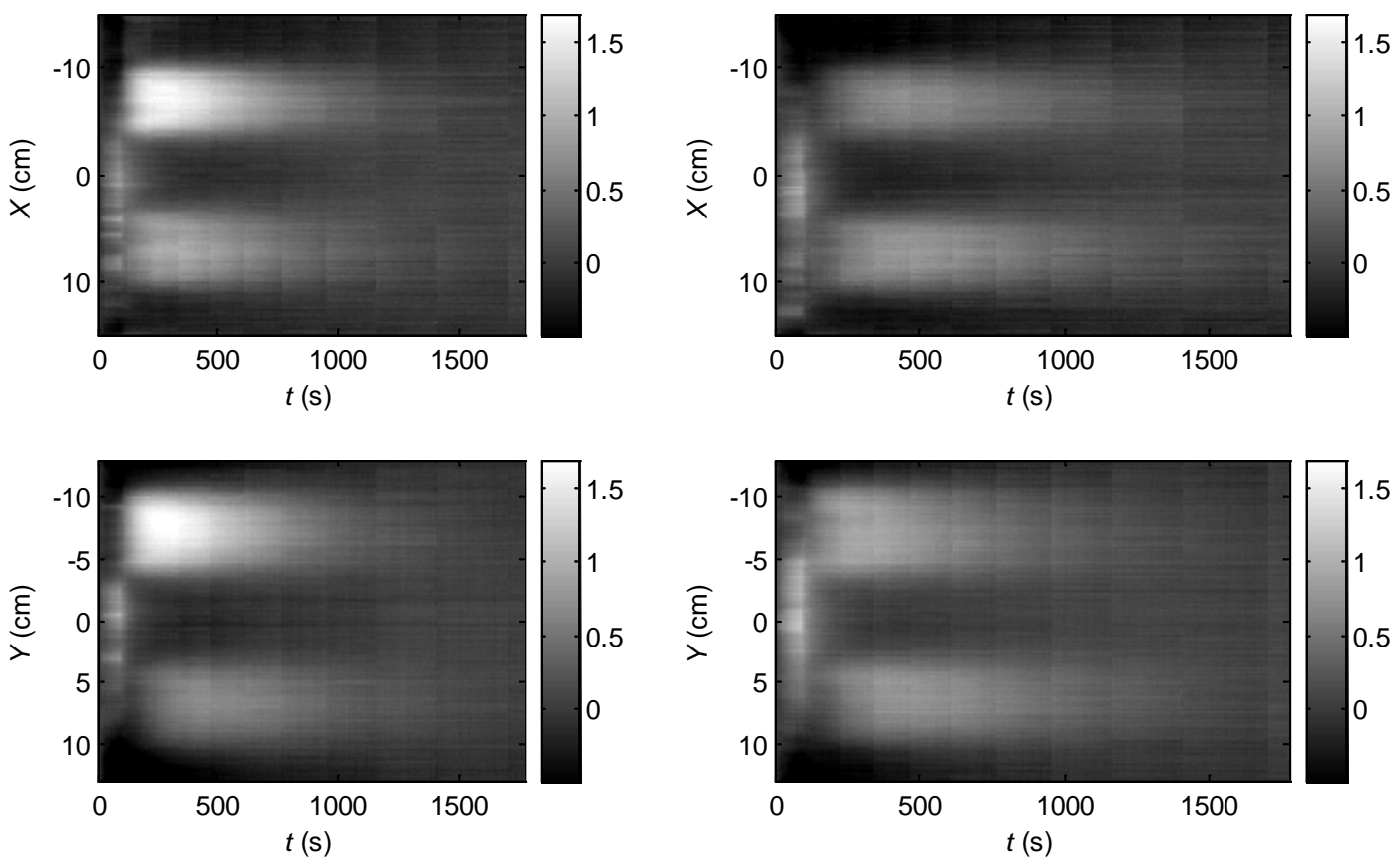

Fig. 14. Absolute contrast computed along horizontal rows 1 (top left) and 2 (top right) and vertical columns 1 (bottom left) and 2 (bottom right); see figure 3 for positions of rows and columns
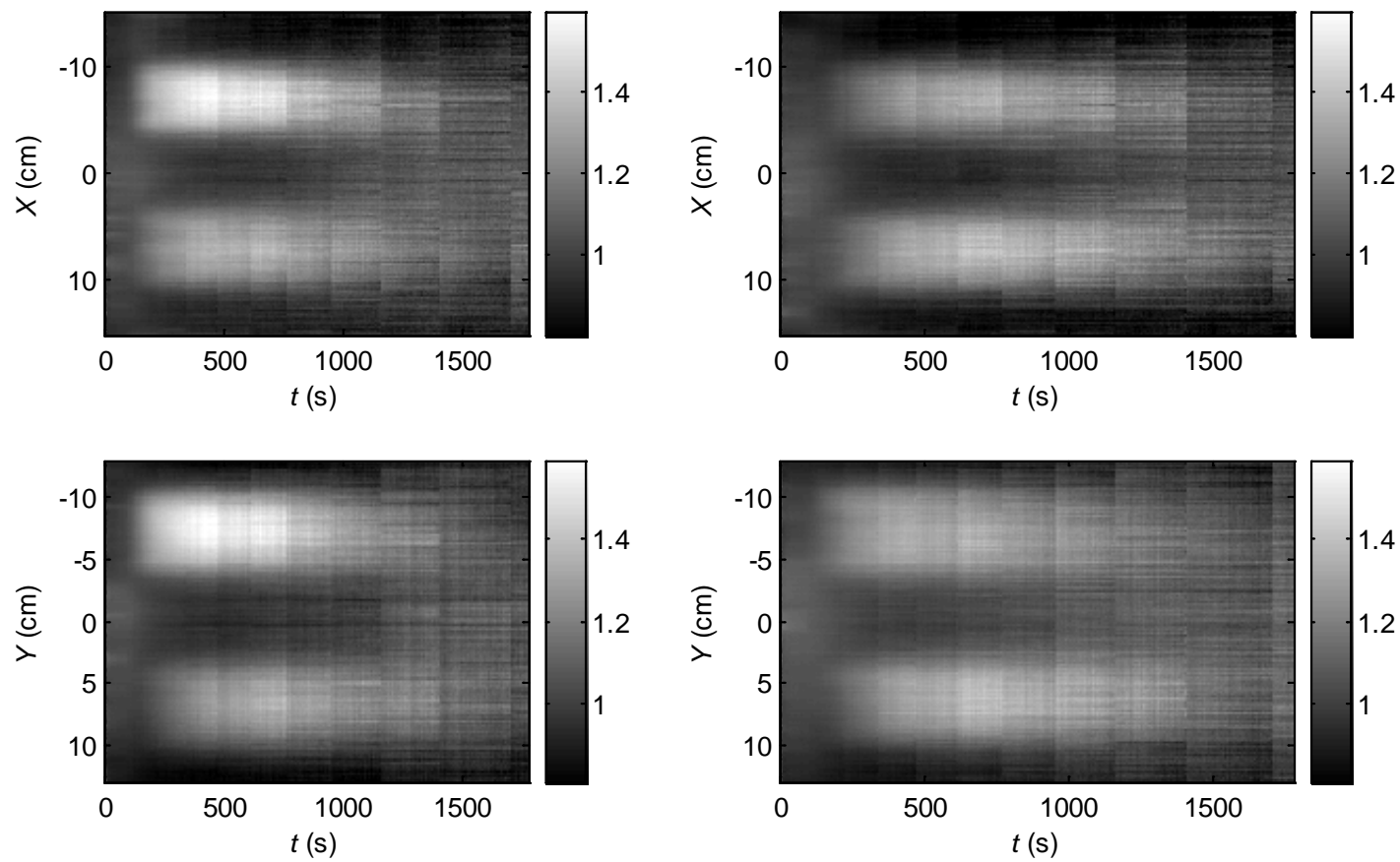

Fig. 15. Standard contrast computed along horizontal rows 1 (top left) and 2 (top right) and vertical columns 1 (bottom left) and 2 (bottom right); see figure 3 for positions of rows and columns

However, the plot of time dependence of absolute contrast obtained from numerical simulations along two horizontal lines passing through the centre of the mosaics allows observing the presence of joints of width greater or equal to $2 \mathrm{~mm}$ (see figure 16 ). 

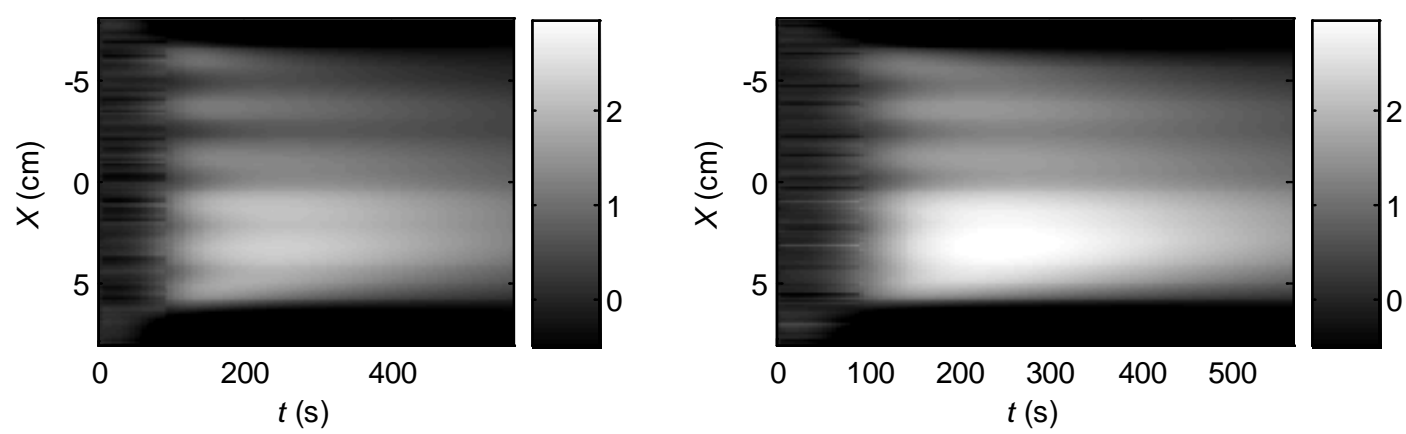

Fig. 16. Absolute contrast computed along horizontal rows; left figure: row crossing mosaic of joints of $4 \mathrm{~mm}$ (top part) and $2 \mathrm{~mm}$ (bottom part) and 2 (top right); right figure: row crossing mosaic of joints of 3mm (top part) and 1 $\mathrm{mm}$ (bottom part)

The maximum temperature difference between joints and polystyrene zones is greater than $0.5^{\circ} \mathrm{C}$ for these zones. The signature of the presence of joints vanishes slowly as the time increases due to 3D diffusion of heat inside the sample.

\subsection{Analysis using singular value decomposition}

Singular value decomposition (SVD) is an interesting tool for the extraction of the spatial and temporal information from a thermographic matrix in a compact or simplified manner. The SVD of an MxN matrix $A(M>N)$ can be calculated as follows ([3],[4]):

$$
\mathbf{A}=\mathbf{U R V}^{T}
$$

where $U$ is an MxN orthogonal matrix, $R$ being a diagonal $N x N$ matrix (with the singular values of $A$ in the diagonal) and VT is the transpose of an NxN orthogonal matrix (characteristic time).

After rearranging the thermograms for every time as columns in A and applying the SVD, the columns of $U$ represent a set of orthogonal statistical modes known as empirical orthogonal functions (EOF) that describe spatial variations of data ([5],[6]). On the other hand, the principal components (PCs), which represent time variations, are arranged row-wise in matrix VT. The first EOF will represent the most characteristic variability of the data; the second one will contain the second most important variability, and so on. Usually, original data can be adequately represented with only a few EOFs.

SVD computations were performed directly on raw sequence of 563 thermal images including the $90 \mathrm{~s}$ of square heating and part of the relaxation phase both for simulation and experiments acquired or generated at a $1 \mathrm{~Hz}$ frequency. The image sequence starts at the beginning of the sample heating. Figure 17 and 18 show results obtain on numerical and experimental data.

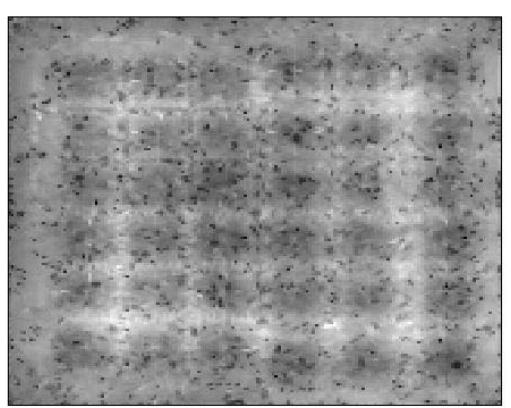

Fig. 17. EOF map calculated with simulated data

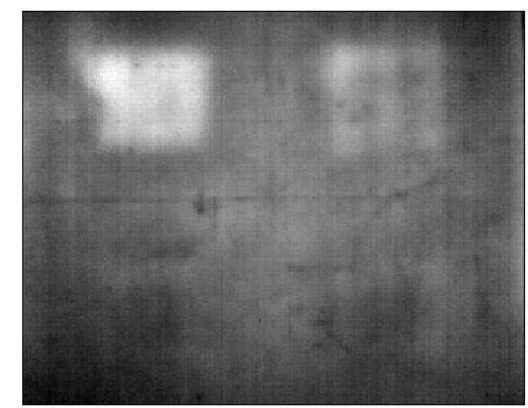

Fig. 18. EOF map calculated with experimental data

For numerical simulation joints and polystyrene parallelepipeds arrangement are discernable on the eof shown in figure 17. For experimental data defect 1 has a clear signature in eof map presented in figure 18 and lower one is observed both for defect 2 and 3. A beginning of discrimination between plaster joints and polystyrene blocks is observed for defect 4.

\subsection{Results discussion}

The noticeable difference in parallelepipeds dimensions between experiments and numerical simulations drives to detection or non detection of joints. Nevertheless, volume ratio for defect composition (i.e. polystyrene and plaster volume versus the total volume) seems to be correlated with detection observed on both experimental and 
numerical data. In Table 2 volume distributions for each defect arrangement both for simulation and experiment are reported.

Table 2. Values of thermophysical properties of plaster, mortar and polystyrene used for numerical simulations

\begin{tabular}{|c|c|c|c|c|}
\hline Plaster joint & \multicolumn{2}{|c|}{ Simulation: $10 \mathrm{~mm} * 10 \mathrm{~mm} * 10 \mathrm{~mm}$ parallelepipeds } & $\%$ plaster & $\%$ polystyrene \\
\hline $1 \mathrm{~mm}$ & Total volume in $\mathrm{m}^{3}$ & 1.02E-05 & $12.11 \%$ & $87.89 \%$ \\
\hline $2 \mathrm{~mm}$ & Total volume in $\mathrm{m}^{3}$ & 1.16E-05 & $22.15 \%$ & $77.85 \%$ \\
\hline $3 \mathrm{~mm}$ & Total volume in $\mathrm{m}^{3}$ & 1.30E-05 & $30.56 \%$ & $69.44 \%$ \\
\hline $4 \mathrm{~mm}$ & Total volume in $\mathrm{m}^{3}$ & 1.44E-05 & $37.67 \%$ & $62.33 \%$ \\
\hline Plaster joint & \multicolumn{2}{|c|}{ Experimentation: $20 \mathrm{~mm} * 20 \mathrm{~mm} * 10 \mathrm{~mm}$ parallelepipeds } & $\%$ plaster & $\%$ polystyrene \\
\hline $1 \mathrm{~mm}$ & Total volume in $\mathrm{m}^{3}$ & 3.84E-05 & $6.35 \%$ & $93.65 \%$ \\
\hline $2 \mathrm{~mm}$ & Total volume in $\mathrm{m}^{3}$ & 4.10E-05 & $12.11 \%$ & $87.89 \%$ \\
\hline $3 \mathrm{~mm}$ & Total volume in $\mathrm{m}^{3}$ & 4.36E-05 & $17.36 \%$ & $82.64 \%$ \\
\hline $4 \mathrm{~mm}$ & Total volume in $\mathrm{m}^{3}$ & 4.62E-05 & $22.15 \%$ & $77.85 \%$ \\
\hline
\end{tabular}

One will observe that the simulation defect with plaster joint of $1 \mathrm{~mm}$ has a volume ratio equivalent to the experimental defect 2, and the $2 \mathrm{~mm}$ one has a volume ratio equivalent to experimental defect 4 . Experimentally, in first approach the defect 2 seems to be at the limit of discrimination between plaster and polystyrene defect arrangement by using absolute contrast time evolution (defect 4 by using SVD). So, looking at this volume ratio evolution and results obtained for simulated data, trends observed experimentally seems to be confirmed. Just the detection but not the mosaic arrangement was obtained with experimental data at the lowest volume ratio. For numerical simulation, mosaic detection and spatial arrangement was obtained by SVD analysis. So, spatial spacing between different mosaics under plaster could have also some influence on detection performances.

\section{Conclusions}

Active infrared thermography was used in square heating configuration to conduct a feasibility study on hidden mosaic detection. Both numerical simulations and laboratory experiments were conducted. Mosaic studied were made of 9 blocks of polystyrene separated by plaster joint of different thickness and covered by $8 \mathrm{~mm}$ plaster layer. Influence of joint plaster thickness onto detection and material mosaic arrangement discrimination was studied. The use of thermal contrast analysis and singular value decomposition for detection allows collecting complementary information on hidden mosaic. First correlation between mosaic material spatial arrangement and detection performances was observed. Numerical simulation partly confirmed this trend. Nevertheless, spatial spacing between each mosaic arrangement will have to be investigated to complete present work. As a perspective, volume ratio distribution approach for mosaic could take benefit of a coupled analysis of mosaic equivalent properties versus its constitutive materials.

\section{REFERENCES}

[1] Maldague X.P.V., "Theory and practice of infrared technology for non-destructive ", Ed. John Wiley \& sons Inc., 2001.

[2] Marchetti M., Ludwig S., Dumoulin J., Ibos L. and Mazioud A., "Active infrared thermography fon non-destructive control for detection of defects in asphalt pavements", Proceedings of QIRT 2008 conference, Krakow.

[3] Rajic N., "Principal component thermography for flaw contrast enhancement and flaw depth characterization in composite structures", Composite Structures, vol 58, pp 521-528, 2002.

[4] Marinetti S., Grinzato E., Bison P. G., Bozzi E., Chimenti M., Pieri G. And Salvetti O. "Statistical analysis of IR thermographic sequences by PCA," Infrared Physics \& Technology vol 46 pp 85-91, 2004.

[5] Ibarra-Castanedo C., Gonzalez D. A., Galmiche F., Bendada A. And Maldague X. P., "Recent Research Developments in Applied Physics On signal transforms applied to pulsed thermography" Recent Research Developments in Applied Physics, vol 9, pp 101-127, 2006.

[6] Dumoulin J., Ibos L., Ibarra-Castanedo C., Mazioud A, Marchetti M., Maldague X. and Bendada A., "Active infrared thermography applied to non emergent defects detection on asphaltic pavement samples: experiments and numerical simulations", Proceedings of 10th international workshop on advanced infrared technology and applications (AITA'09), Florence (Italy), 8-11 september 2009. 